\title{
The Research and Application of Flipped Classroom Bleed into CDIO
}

\section{Shanshan $\mathrm{Li}^{1, \mathrm{a}}$, Hui Wang ${ }^{1, \mathrm{~b},{ }^{*}}$ and Xiangtao Chen ${ }^{1, \mathrm{c}}$}

1 The Network Information Center of Henan University of Science and Technology, Luoyang,

Henan, Zhongguo

alss@haust.edu.cn, bwh@haust.edu.cn, ccxt@haust.edu.cn

*Hui Wang

Keywords: Mode of education, Flipped classroom, CDIO.

\begin{abstract}
As a new mode of education, flipped classroom gets popular attention both at home and abroad in recent years. By reversing the process of delivering and expanding knowledge, the flipped classroom changes the traditional teaching structure, and takes advantage of the time for face-to-face communication to innovate teaching methods. In order to better applied in the teaching, combining teaching practice, introducing the CDIO engineering education concept, build a new mode of flipped classroom bleed into CDIO, its practical effect has carried on the preliminary verification, by introducing the CDIO concept, the new model of flipped classroom has better realized the knowledge transfer, internalization and consolidated. Practiced the new model in the specific teaching process of computer organization and gained remarkable achievements.
\end{abstract}

\section{融入CDI0理念的翻转课堂教学模式研究和应用}

\author{
李姗姗 ${ }^{1, a}$, 王辉 $2, b,{ }^{*}$, 陈祥涛 $3, c$ \\ 1河南科技大学网络信息中心, 洛阳, 河南, 中国 \\ alss@haust.edu.cn, bwh@haust.edu.cn, ccxt@haust.edu.cn \\ *王辉
}

关键词：教育模式；翻转课堂; CDI0

中文摘要. 作为一种新兴的教育模式, 翻转课堂近几年在国内外颇受关注。通过对教学结构 的颠倒安排, 翻转课堂改变了传统教学中的师生角色, 并对传统课堂的时间进行了重新规划, 革新了传统的教学模式。为了使翻转课堂更好的应用于教学, 结合教学实践, 引入 “CDIO” 工程教育理念, 构建了融入CDIO理念的翻转课堂新模式, 该模式用 CDIO理念来指导翻转课 堂课前、课中及课后活动的设计, 更好的实现了翻转课堂中知识的传递、内化和巩固。将该 模式应用于计算机组成原理的课堂教学中，效果显著。

\section{1. 引言}

翻转课堂是基于现有的信息技术手段，构建信息化教学环境，并重新规划课前、课中和 课后的教学活动, 颠覆了人们对传统课堂模式的思维习惯, 从新的角度揭示课堂的新形式、 新内含 ${ }^{[1]}$, 使课堂教学从以教师讲解为中心转变为以学生课堂互动为中心, 教师从教学过程

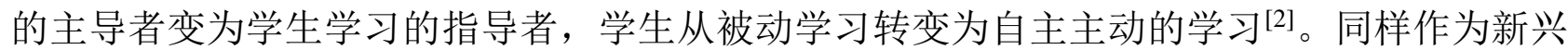


的教育理念, CDIO教育理念和翻转课堂在一定程度上存在相呼应的切合点, 将二者相结合有 利于开拓翻转课堂的新思路。

\section{CDIO}

CDIO工教育模式是由麻省理工学院和瑞典皇家工学院等四所大学创立的, 它代表构思 （Conceive）、设计（Design）、实现（Implement）和运作（Operate），是以产品研发到产 品运行的生命周期为载体, 让学生以主动的、实践的、课程之间有机联系的方式学习工程 ${ }^{[3-4]}$, 它包括了三个核心文件: 1 个愿景, 为学生建立在真实世界的产品和系统的构思一设计一实现 一运行过程的教育环境; 1 个大纲, 将工程毕业生的能力分为工程基础知识、个人能力、人际 团队能力和工程系统能力四个层面, 使工程教育改革具有更加明确的方向性; 12条标准, 对 CDIO工程教育模式的实施和检验进行了系统的、全面的指引, 对学生和教师都具有重要指导 意义 ${ }^{[5]}$ 。迄今为止, 已有几十所世界著名大学引入了 $\mathrm{CDIO}$ 教育理念, 取得了良好效果。

\section{3. 翻转课堂教学模式}

学习者的学习过程大体上分为两个步骤, 即知识的传递和知识的内化。传统的教学模式 是通过教师面对面的课堂讲授来完成知识的传递, 学生通过课后作业或实践来完成知识的内 化, 由于学习者个体的差异, 导致教师在课堂的有限时间内不能照顾到所有的学生, 更不能 掌握学生在课下的学习状况。而近几年在美国高校教学过程中比较流行的翻转课堂教学模式 是对这种传统教学模式的颠覆, 所谓翻转课堂, 就是在信息化环境中, 课程教师提供以教学 视频为主要形式的学习资源, 学生在上课前完成对教学视频等学习资源的观看和学习, 师生 在课堂上一起完成作业答疑、协作探究和互动交流等活动的一种新型的教学模式 ${ }^{[6]}$ 。翻转课 堂与传统课堂的教学结构比较如下图所示:

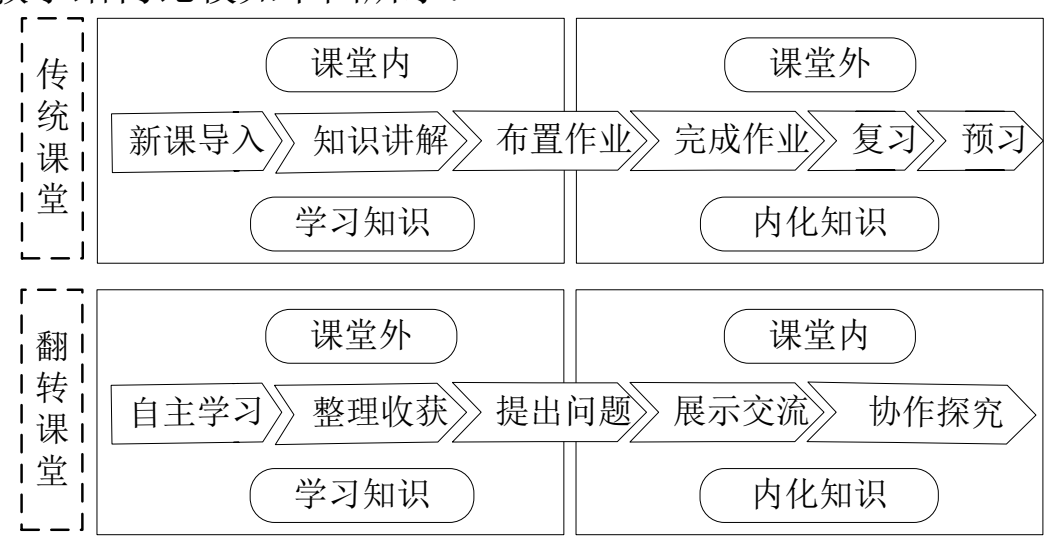

图1 传统课堂与翻转课堂的教学结构比较

由图中可以看出, 翻转课堂是对传统课堂教学形式的逆转, 两者的教学过程有着本质的 不同。传统课上的概念讲解、意义理解在翻转课堂中由学生自主探究和建构, 而传统课下的 知识应用在翻转课堂中则由学生在教师的指导下进行, 即传统课堂学习知识在课内, 内化知 识在课外, 而翻转课堂则正好相反, 学习知识在课外, 内化知识在课内 ${ }^{[6]}$ 。翻转课堂的教学 方式旨在为学生建立一种混合式学习环境，包括采用任何技术来提升课堂学习效果，使教师 能够在课堂上抽出更多的时间与学生互动交流, 而不是将课堂时间用于传统意义上的讲课, 它的实现方式是让学生利用课外时间观看并学习由授课教师制作的或推荐的教学视频资源以 及授课材料, 把课堂时间用来问题解决或专题研究 ${ }^{[7]}$, 这样即能增加学生在教师指导下亲身 实践的时间, 也能通过内化知识帮助学生产生新的想法, 同时还能满足学生个性化辅导的需 求。 


\section{4. 融入CDIO理念的翻转课堂教学模式设计}

翻转课堂通常以文档、PPT和视频作为学生课前学习的资料, 学生通过自主的方式进行 学习, 但这种单纯的视频文字方法的学习效率并不高, 这使得学习材料所产生的学习效果并 不尽如人意, 想要提高学生课前知识的准备效果就要融入做和用的成份。因此, 可以将学生 课前的自主的学习转变为完成任务的形式学习, 而不是单纯地观看文档视频等, 这样在课前 阶段就促使学生运用实际演练方法, 为提高自主学习的效果奠定基础。在课堂上进行评价交 流时, 学生将自己完成任务的创新点和自己认为比较有特点的地方展示给教师和其他的同学 们, 积极地论述自己的观点和创新点, 这也就进一步提高了学习的效果。为此, 根据翻转课 程教学模型的特点和 CDIO教育模式的能力要求, 设计了以下融入CDIO理念的翻转课堂教学 新模式，如下图所示:

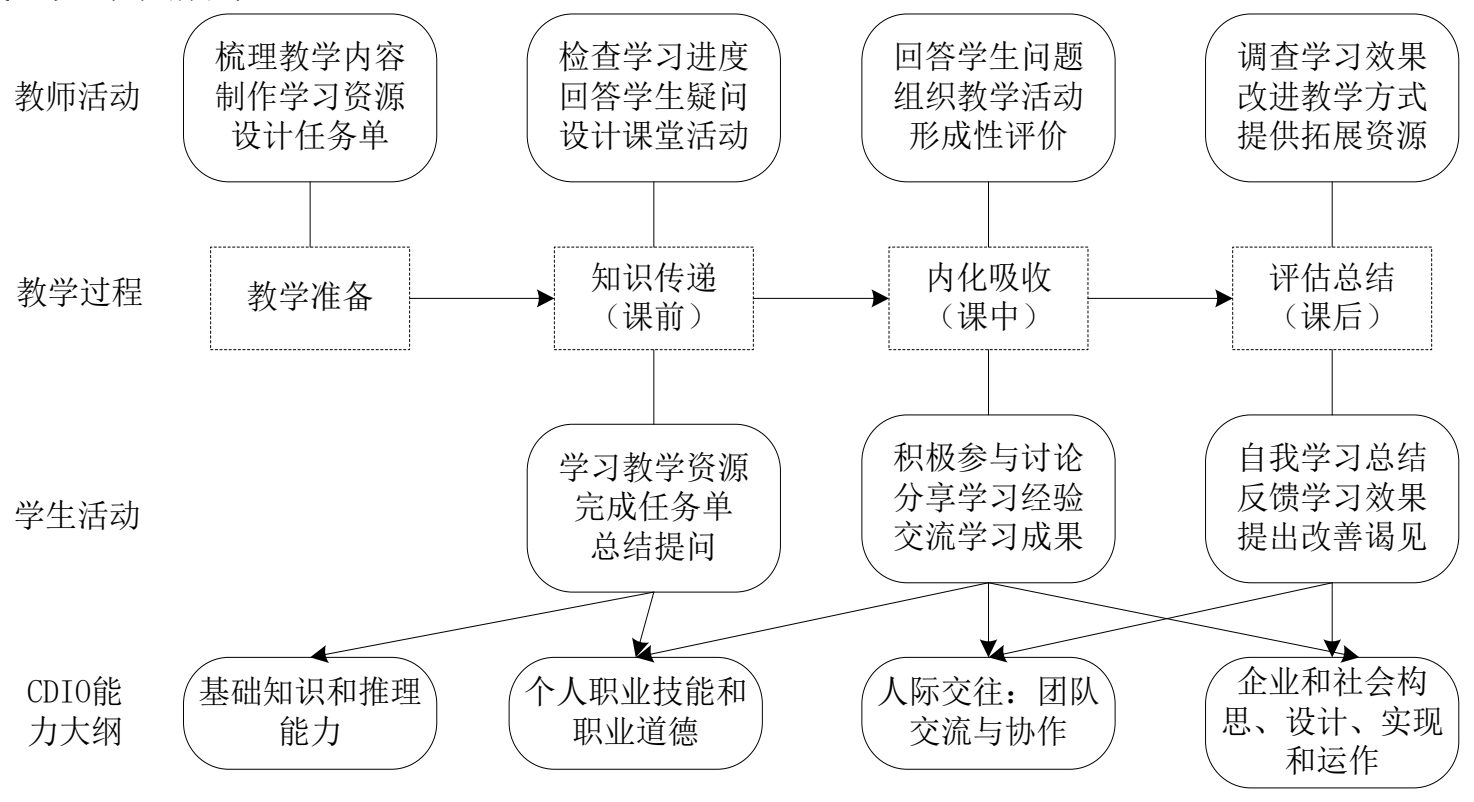

图 2 融入 CDIO 理念的翻转课堂教学模型

从模型图中来看, 翻转课堂的每个阶段与 CDIO的能力大纲很好的相呼应, 新的模型图中 翻转课堂的学习共分为以下几个阶段。

1. 教学准备阶段

由于翻转课堂知识传递主要是通过视频、文档、PPT等方式进行, 在进行翻转课堂学习 之前, 教师需要开发教学资源以供学习者学习, 因此无论是CDIO的教学理念, 还是翻转课堂 的教学模式, 都对教师的专业能力提出了新的、更高标准的要求。在该阶段, 教师首先要根 据教学安排的需要, 重新梳理单元教学内容, 把适合通过教师讲授、演示的内容分离出来, 主要的工作有: 明确课程的知识点和教学要求, 剖析知识点或技能要点, 整理其相互关系; 明确学习者的起点水平、学习动机以及一般特征; 确定教学资源、教学策略, 编写教学过程; 根据学习目标设计学习任务卡; 制作课件和教学视频。

2. 知识传递阶段

知识的传递过程是学生进行自主学习的过程, 根据教师下发的学习任务单, 学生按照要 求进行课前学习, 首先要查看学习任务单, 明确任务单上的学习任务; 然后根据任务单上的 要求观看视频, 如遇到问题可以记录下来; 学习完之后进行习题测试, 在此期间如遇问题可 以在线与教师和其他同学共同讨论。这个过程中, 一方面学生可以自由选择学习资源、自主 掌握学习进度, 满足个性化学习需求, 另一方面教师通过批改学生学习任务的完成情况, 分 析在线答疑和讨论情况, 有利于获取课前的学习效果并发现学生的问题。该阶段是基于学生 
自己的思考解决问题, 主动学习方法在CDIO环境下促进专业目标的实现, 主要培养学生基础 知识和推理能力、个人职业技能和职业道德等。

\section{3. 内化吸收阶段}

本阶段的首先是解决课前知识传递阶段教师搜集到的问题，根据问题的类型可以组织探 究式或讨论式课堂。在完成问题解答后, 教师设置其他典型问题, 根据问题的难易程度组织 学生进行自主探究或小组协作学习。活动进行过程中, 教师引导学生运用所学知识解决问题, 调动学生参与的积极性, 对于各小组解决不了的问题进行个性化辅导, 学生要主动配合教师 积极主动参与活动, 踊跃发表观点, 主动反馈活动进展情况和遗留问题, 增加与教师的交流 互动, 并且小组间要主动分享学习经验, 交流学习成果, 以实现知识的建构和内化, 主要培 养学生的团队协作和交流技能以及工程系统能力。

\section{4. 课后评估总结}

教学活动结束后, 教师根据学生的完成情况进行点评, 学生配合教师对教学效果进行总 结。学生要撰写学习总结, 回顾课上和课下整过学习过程, 记录学习感悟, 反馈学习效果, 并提出改进意见, 教师需要总结学生们反馈的学习效果和改进意见, 找出教学过程中存在的 不足, 并修改教学过程。教师还需要收集与教学内容相关的拓展学习资源设置拓展任务, 有 兴趣的同学可以挑战拓展任务, 实现学生对所学知识的巩固和拓展。

从融入CDIO理念的翻转课堂教学模型来看, 一方面 CDIO能力大纲可以为翻转课堂的教 学提供目标依据, 另一方面可用CDIO的理念来指导翻转课堂的教学活动设计, 两者的结合能 更好的促进传统教学模式的改革。从新教学模式的实施过程来看, 整个教育过程更加的人性 化, 学生是主体, 可以自由掌握学习时间、学习进度, 并可通过各种方式与同学之间进行交 流互动, 对于同学间解决不了的问题可以远程反馈给老师, 这是一种人性化的学习策略, 能 够使学生自己体会到学习的重要性并能找到存在感, 能够有效的改善学生的学习效果。教师 在此过程中运用新的策略进行教学活动的设计, 首先根据学生的特点进行分组, 根据学生的 兴趣分配各类讨论的题目, 让学生拥有课堂话语权, 可随时提出自己的观点和想法, 教师随 时捕捉各小组的动态并及时的给予指导, 在这个过程中使学生和教师成为了密切的学习伙伴, 构建了和谐的师生关系。

\section{5. 融入CDIO理念的翻转课堂教学模式实践研究}

为检验融入CDIO理念的翻转课堂教学模式的可行性, 将该教学模式应用于计算机组成原 理的课堂中, 开展融入CDIO理念的翻转课堂的实证研究。“计算机组成原理”是计算机科学与 技术专业本科教学的一门专业基础课程, 设置这一课程的目的是, 使学生了解和掌握计算机 系统的硬件组成和基本工作原理, 了解计算机硬件各主要部件的结构、原理和设计方法。该 课程的内容显得有些枯燥, 用传统的课堂教学模式效果不是很好, 因此, 将新的教学模式应 用于该门课程的教学中, 具体的应用实践如下:

\section{1 实施过程}

(1)教学准备阶段: 在计算机组成原理开课前, 我们将课程内容按知识点做了仔细的梳理 和划分, 每个知识点都录制了10分钟左右的学习视频, 并制作了相应的学习任务单, 根据一 学期的课时安排, 按时的给学生发放学习任务单。为了使学生的学习过程有效可控, 选择了 目前主流的网络学习平台, 将课程的视频、文档等资源全都上传到课程平台上, 并且学生的 作业、测验也通过该平台进行，使学生的整个学习过程有迹可寻。

(2)知识传递阶段：学生在接受到学习任务之后，根据任务单上的要求，自主选择时间来 学习平台上提供的各类资料, 并完成相应的作业, 遇到自己解决不了的问题, 学生可在学习 平台的讨论区中提出, 其他同学或老师都可进行回答或与其讨论, 通过这种学习模式, 不但 学到了老师要求的学习任务, 也了解了其他同学的思维模式。即初步构建了学习的内容, 也 有利于学生学习能力的提高。 
(3)内化吸收阶段: 此阶段是在课堂上完成的, 首先, 教师根据网上记录的学生学习及讨 论情况, 重点讲解学生学习过程中有困难的知识点, 加强学生的理解; 然后根据章节内容设 置相应的讨论题, 分小组进行讨论, 老师在一旁进行指导, 讨论结束后, 各小组派一名学生 汇报讨论的结果。在这一过程中, 进一步构建了所学的知识, 也让学生积极的参与到小组的 讨论中，即锻炼了学生的交流互动能力，也培养了其团队责任感。

(4)课后评估总结阶段: 在实行翻转课堂时, 评估总结应该从多维度、多方式进行, 对学 生的评估不仅仅由老师一个人完成, 还应该学生自己及其他的学生, 评估的内容包括学习平 台上记录的学习及讨论情况, 课堂中学生的提问、分析、解决及汇报问题的情况等, 另外学 生也可根据上一次的评估情况来制定下一步的教学计划, 规划下一次的教学活动。

\section{2 实施效果}

融入CDIO理念的翻转课堂教学模式激发了学生的学习兴趣, 提高了学生自主学习、协作 学习和动手实践的能力, 增强了独立发现问题、分析问题和解决问题的能力, 教学效果提高 明显。在采用新模式教学一个学期之后, 我们对授课学生进行了问卷调查, 主要是从对融入 CDIO理念的翻转课堂教学模式的满意度、对该模式教育下学习效果的满意度、对该模式教育 下自身各种能力提高的满意度等。具体调查结果如下图所示:

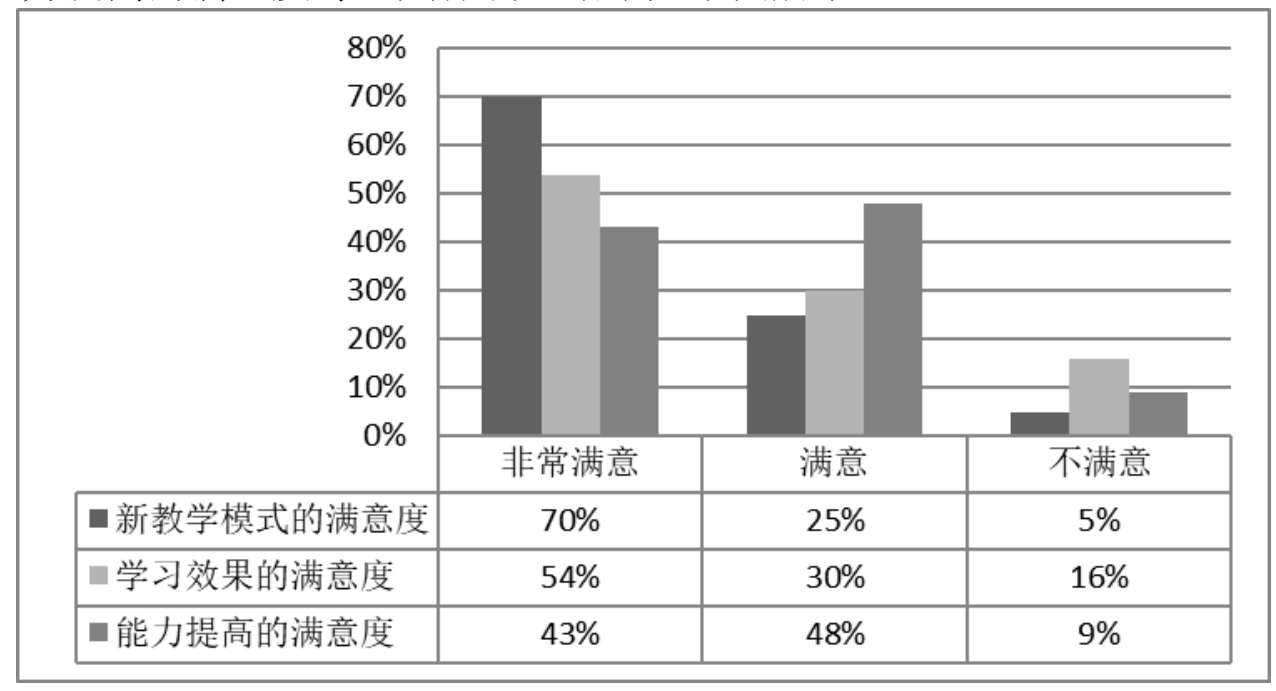

图3 融入CDIO的翻转课堂实施效果满意度分布

从调查结果来看, 学生对融入CDIO理念的翻转课堂教学模式的满意度中, 绝大部分学生 都能很好的适应该模式的教学, 只有 $5 \%$ 的学生不适应, 对于这些学生, 教师可进行个别的指 导性学习, 使其尽快适应该模式的教学; 在学生对该模式实施效果后学习结果的满意度中, 通过该模式的实施绝大部分同学都获得了较好的学习效果, 仍有 $16 \%$ 的同学不满意, 教师可 针对这些同学进行谈话, 获得他们理想的学习效果, 有好的意见可进行采纳对新的教学模式 进行适当的改进; 在该模式实施下学生各种能力提高的满意度中，有 $9 \%$ 的同学认为自己的能 力没有提高, 教师可与这些学生进行交流, 帮助其找出原因。从总体上看, 融入CDIO理念的 翻转课堂教学模式实施效果良好。

\section{6. 结束语}

翻转课堂作为新的课堂教学模式, 也需要不断地创新和发展。本文引入CDIO的教育理念, 提出了融入CDIO理念的翻转课堂教学模式, 可以使学习效率在已有的翻转课堂模式下得到进 一步的提高, 更好地提升学生的参与度。将“CDIO”与“翻转课堂”相结合, 学生在学习任务单 的指引下有了更大的学习自由度, 学生可以根据自身的情况更好的安排学习, 更有利于不同 层次学生的个性化学习, 实践证明, 大部分学生对新教学模式的实施比较满意。 


\section{致谢}

本文为河南省高等学校重点科研项目计划“MOOC环境下高校资源整合与共享的实现路 径研究” (项目编号：17B630004) 、河南省高等学校优质数字化教育资源云共享模式与机制 研究（编号：15A630002）及河南省教育科学”十二五“规划2015年度课题“融合“CDIO”理念的 翻转课堂教学模式研究（项目编号：[2015]-JKGHYB-0630）的阶段性成果之一。

\section{References}

[1] Fuhai. An, Flipping the classroom: from "timing reconstruction" to "deep learning", Educational Science Research, vol.3, pp. 71-75, 2018.

[2] Xueyan Zhao. Reality and Virtuality: Analysis of Classroom Change in Flipping Classroom, Basic education research, vol.23, pp. 129-131, 2018.

[3] John M. The application of CDIO Standards in the evaluation of Swedish engineering degree programmers[J]. World Transaction on Engineering and Technology Education, vol.5, pp. 361-364, 2006.

[4] Jianzhong. Zha. On CDIO Model under"Learning by Doing" Research in Higher Education of Engineering, vol.3, pp. 1-9, 2008.

[5] Crawley E F. Creating the CDIO Syllabus, a universal template for engineering education[C]// 32nd Annual Frontiers in Education, vol. 3, pp. 8-13, 2002.

[6] Zhaojie Hao, Hao Li, and Liming Ma, Flipping the classroom to the classroom teaching reform in colleges and universities: value, limit and transcendence, China Education Info, vol. 18, pp. $1-6,2018$.

[7] Chunhua Wei, Design and Practice of Flipping Classroom Teaching Model Based on Micro-Lessons_- Taking Internet Finance Course as an Example, European Journal of Journal of Higher Education, vol. 8, pp. 105-107, 2019. 